\title{
Chiral doublings of heavy-light hadrons: New charmed mesons discovered by BABAR, CLEO and BELLE
}

\author{
Maciej A. Nowak \\ M. Smoluchowski Institute of Physics, Jagiellonian University, \\ 30-059 Kraków, Reymonta 4, Poland \\ e-mail:nowak@th.if.uj.edu.pl
}

\begin{abstract}
We remind the chiral doubling scenario[1, 2] for hadrons built of heavy and light quarks. Then we recall the arguments why new states $D_{s}(2317), D_{s}(2460), D_{0}(2308)$ and $D_{1}^{\prime}(2427)$ should be viewed as chiral partners of $D_{s}, D_{s}^{*}, D$ and $D^{*}$, respectively. We summarize with the list of predictions based of chiral doubling scenario for other heavy-light hadrons.
\end{abstract}

Recently, experimental physics of hadrons with open charm has provided several spectacular discoveries:

- First, BaBar [3] has announced new, narrow meson $D_{s J}^{*}(2317)^{+}$, decaying into $D_{s}^{+}$ and $\pi^{0}$. This observation was then confirmed by CLEO [4], which also noticed another narrow state, $D_{S J}(2463)^{+}$, decaying into $D_{s}^{*}$ and $\pi^{0}$. Both states were confirmed by Belle [5], and finally, the CLEO observation was also confirmed by BaBar [6]. From the moment of discovery, both states triggered a flurry of activity among the theorists. Experimental results were surprising, since such states were neither expected below the $D K$ and $D * K$ thresholds nor were expected to be that narrow. Till today, several theoretical constructions were proposed to explain the masses, quantum numbers and decay patterns, most of them discussed during this conference.

- Second, Belle has not only measured the narrow excited states $D_{1}, D_{2}$ with foreseen quantum numbers $(1+, 2+)$, but provided also first evidence for two new, broad states $D_{0} *(2308 \pm 17 \pm 15 \pm 28)$ and $D_{1}^{\prime}(2427 \pm 26 \pm 20 \pm 17)$. Both of those are ca $350-400$ $\mathrm{MeV}$ higher above the usual $D_{0}, D^{*}$ states and seem to have opposite parity.

- Third, Selex has provided preliminary data for doubly charmed baryons [7]. On top of known since December $c c d$ state (3520), four other cascade $j=1 / 2$ states are visible. Their masses are a challenge for standard estimations based on potential models [8]. This calls for alternative predictions, based either on chiral solitonic models or diquark scenarios.

In this talk, we recall that actually the presence of this type of states was predicted by theoretical arguments already in 1992 and 1993, and is in fact required from the point of view of symmetries of the QCD interactions. The two, mentioned above particles observed by BaBar and CLEO are the first, theoretically anticipated [1, 2] chiral partners of hadrons built out of light and heavy quarks. As such, they should represent rather a pattern of spontaneous breakdown of chiral symmetry than isolated events. 
Strong interactions involve three light flavors $(\mathrm{u}, \mathrm{d}, \mathrm{s})$ and three heavy flavors $(\mathrm{c}, \mathrm{b}$, t) with respect to the QCD infrared scale. The light sector (1) is characterized by the spontaneous breaking of chiral symmetry, while the heavy sector (h) exhibits heavyquark (Isgur-Wise) symmetry [9]. In our original work [1] we addressed the question of the form of the heavy-light effective action in the limit where light flavors are massless, while the heavy flavors are infinitely massive. The novel aspect of our original derivation was that consistency with the general principles of spontaneously broken chiral symmetry requires the introduction of chiral partners in the form of a $\left(0^{+}, 1^{+}\right)$ multiplet of pseudoscalars and transverse vectors [1]. In the heavy-quark limit, the splitting between the chiral partners is small and of the order of the "constituent quark mass". The chiral corrections to the splitting were recently shown to be of order $m_{\pi}^{2} / 4 m_{h}$, and therefore small irrespective of an effective Lagrangian analysis [10].

In brief, to leading order in the heavy-quark mass, the one-loop effective action for the chiral doubler $\left(0^{+}, 1^{+}\right)$

$$
G=\frac{1+\psi^{\prime}}{2}\left(\gamma^{\mu} \gamma_{5} \tilde{D}_{\mu}^{*}+\tilde{D}\right)
$$

duplicates the known action [11] for the standard $\left(0^{-}, 1^{-}\right)$multiplet

$$
H=\frac{1+y^{\prime}}{2}\left(\gamma^{\mu} D_{\mu}^{*}+i \gamma_{5} D\right) \text {. }
$$

i.e.

$\mathscr{L}^{G}=-\frac{i}{2} \operatorname{Tr}\left(\bar{G} \nu^{\mu} \partial_{\mu} G-v^{\mu} \partial_{\mu} \bar{G} G\right)+\operatorname{Tr} V_{\mu} \bar{G} G v^{\mu}-\mathbf{g}_{G} \operatorname{Tr} A_{\mu} \gamma^{\mu} \gamma_{5} \bar{G} G-\mathbf{m}_{\mathbf{G}}(\Sigma) \operatorname{Tr} \bar{G} G$

The axial $A_{\mu}$ and vector $V_{\mu}$ currents are light currents, contributing to transitions in odd or even number of pions, respectively (or generically, $S U\left(N_{l}\right)$ Goldstone bosons). The key difference is the opposite sign in the sign of the constituent mass contribution in (3), with respect to similar term for $H$ multiplet. The sign flip follows from the $\gamma_{5}$ difference in the definition of the fields $H$ and $G$. In other words: it is sensitive to the parity content of the heavy-light field since $H v=-H$ and $G k=+G$. The result is a split between the heavy-light mesons of opposite chirality. This unusual contribution of the chiral quark mass stems from the fact that it tags to the velocity $H x \bar{H}$ of the heavy field and is therefore sensitive to parity. The reparametrization invariance (invariance under velocity shifts of the heavy quark to order one) introduces mass shifts that are parity insensitive to leading order in $1 / m_{h}$ [12]. Chiral partners communicate with each other via light axial currents

$$
\mathscr{L}_{H G}=\sqrt{\frac{\mathbf{g}_{G}}{\mathbf{g}_{H}}} \operatorname{Tr}\left(\gamma_{5} \bar{G} H \gamma^{\mu} A_{\mu}\right)-\sqrt{\frac{\mathbf{g}_{H}}{\mathbf{g}_{G}}} \operatorname{Tr}\left(\gamma_{5} \bar{H} G \gamma^{\mu} A_{\mu}\right)
$$

with no vector mixing because of the parity.

We visualize chiral doublers scheme for mesons in the form of cartoon, see Fig. 1. The three-dimensional "cube" is aligned along three "directions":

- chiral symmetry breaking (horizontal, green) 


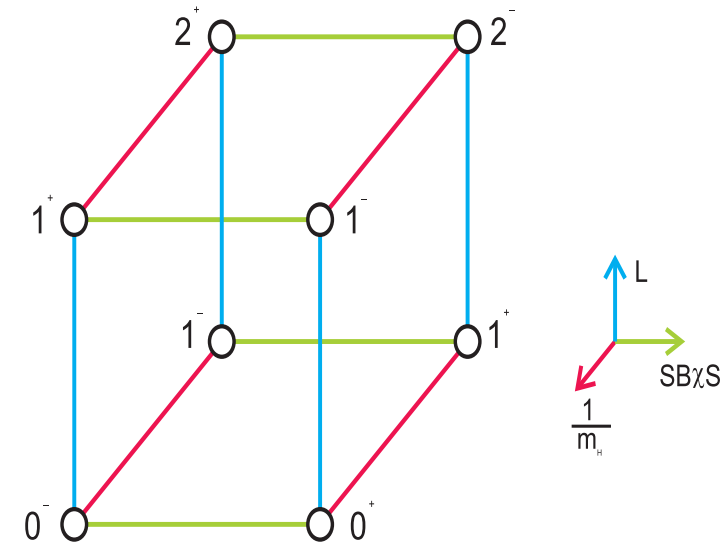

FIGURE 1. Cartoon representing schematic classification of chiral doublers.

- Isgur-Wise symmetry breaking (skew, red)

- total light angular momentum (vertical, blue).

The corners of the cube represent generic $h \bar{l}$ mesons, i.e. we expect similar "cubic" patterns for $c \bar{s}, c \bar{u}, c \bar{d}, b \bar{s}, b \bar{u}, b \bar{d}$ mesons. Let us focus on $c \bar{s}$ states, i.e $D_{s}$-cube. Lower left rung represents known pseudoscalar $0^{-} D_{s}(1969)$ and vector $1^{-} D_{s}^{*}(2112)$, belonging to $j_{l}=1 / 2$ light angular momentum representation. The splitting between them is an $1 / m_{c}$ effect and is expected to vanish in infinitely heavy charm quark limit, i.e. both particles would have form the $H$ multiplet. The upper left rung corresponds to $j_{l}=3 / 2$ representation, i.e. $1^{+}$and $2^{+}$excited multiplet. Here $D_{s 1}(2536)$ and $D_{s J}^{*}(2573)$ are the candidates, separated by (smaller for excited states) $1 / m_{c}$ origin mass splitting. Similar pattern applies for the non-strange charmed mesons (D-cube), i.e. $D(1865)$, $D^{*}(2010), D_{1}(2420)$ and $D_{2}(2460)$. This "left plaquette" of the cube completes the standard, "pre-BaBarian" charmed meson spectroscopy.

The novel aspect is the existence of the chiral doublers, i.e. the appearance of the right plaquette. First, we expect two chiral partners for $D_{s}$ and $D_{s}^{*}$, representing right lower rung. Here newly discovered $D_{S J}^{*}(2317)$ and $D_{S J}(2463)$ are the candidates for the $\left(0^{+}, 1^{+}\right)$scalar-axial $G$ multiplet. The averaged splitting for $\left(0^{+}, 0^{-}\right)$and the averaged splitting for $\left(1^{+}, 1^{-}\right)$are $349.2 \pm 0.8$ and $346.8+1.1$, respectively, i.e. almost identical, as predicted a decade ago [1, 2]. Naturally, the splitting within the $G$ multiplet, i.e. between the masses of the new BaBar state and CLEO state, is identical to the splitting between the $\left(1^{-}, 0^{-}\right)$pair. The narrowness of the new states is basically the consequence of the kinematic constraints, as pointed by [13]: since the chiral split is smaller than the mass of the kaon, these states live longer. On top of this effect, the isospin conservation most probably forces the pionic decay via virtual $\eta$ decay, suppressing the rate even further [13]. Electromagnetic transitions, estimated on the basis of chirally doubled lagrangians in [13] are also in agreement with the experimental data. It is noteworthy to stress, that chiral Ward identities additionally constraint the amplitudes of the pionic decays for the $H$ multiplets, $G$ and for $G-H$ pionic transitions [10].

Let us move now towards the excited states. On the basis of the chiral doublers scenario, we would also expect the chiral partners for the excited $j_{l}=3 / 2$ multiplet, i.e. new chiral pair $\left(1^{-}, 2^{-}\right)$[12]. Alternatively, this pair could be also viewed as the 
$j_{l}=3 / 2$ excitation of the BaBar-Cleo $\left(0^{+}, 1^{+}\right)$multiplet. Our prediction for the masses of this new pair reads:

$$
\begin{aligned}
& m\left(\tilde{D}_{s 1}\right)=2721 \pm 10 \mathrm{MeV} \\
& m\left(\tilde{D}_{s 2}\right)=2758 \pm 10 \mathrm{MeV}
\end{aligned}
$$

where we used [10] as an input the observed BaBar and CLEO splitting for the chiral multiplet $\left(0^{+}, 1^{+}\right)$and the mass formulae obtained in [12]. Note that the chiral splitting for excited states is approximately half of the chiral splitting for the ground pair.

This completes the identification of corners of the "cube". Left and right plaquettes are chiral copies, front and back plaquettes become degenerate in infinite mass of the heavy quark and the lower and upper plaquettes are separated by the excitation of total light angular momentum $j_{l}$. We do not discuss here the possibility of even higher angular excitations, i.e. additional $j_{l}=5 / 2$ plateau, pointing only that first such states $\left(2^{-}, 3^{-}\right)$ may naturally appear above $3 \mathrm{GeV}$.

Let us move now towards non-strange charmed mesons. Here two states from Belle, $D_{0}^{*}(2308)$ and $D_{1}^{\prime}(2427)$ are natural candidates for lower right rung of the D-cube, i.e. for the chiral doublers of $D(1825)$ and $D^{*}(2010)$. There are however broad, since neither kinematic nor isospin restrictions apply here, contrary to their strange cousins.

One of the arguments against the interpretation that the above pair might be a chiral doubler is based on the values of the "chiral" mass shift, which (modulo experimental errors) seems to be equal of even larger for the non-strange mesons than for the strange ones. Actually, this argument acts rather in favor for the chiral doublers scheme [10]. A simple parametrization for the constituent quark mass (with good comparison to instanton liquid model and lattice data) was quoted in [14]

$$
\Sigma\left(m_{l}\right) \approx m_{l}+\Sigma(0)\left(\sqrt{1+\left(m_{l} / d\right)^{2}}-m_{l} / d\right)
$$

with $\Sigma \approx 345 \mathrm{MeV} / c^{2}, d \approx 198 \mathrm{MeV}$. For a strange quark mass $m_{s} \approx 150 \mathrm{MeV}$, the second term is reduced to $\Sigma(0) / 2$, making the combination (6) weakly dependent on $m_{l}$ and of order $\Sigma$ all the way up to the strange quark mass. Thus, both mass splittings are about the same for $(u, d, s)$ heavy-light mesons. Taking e.g. the value $\Sigma(0)=400$ $\mathrm{MeV}$ one could easily estimate the effects of chiral splitting for strange quark to be of order $350 \mathrm{MeV}$, so even smaller than for non-strange quarks. We would like to mention, that several simplified models just add the constant chiral dressing to the current mass, ignoring the feedback of the explicit breaking of the chiral symmetry on the vacuum effects.

Let us mention for completeness, that the chiral doubling should be even more pronounced for bottom mesons, since the $1 / m_{h}$ corrections are three times smaller. For $m_{s}=150 \mathrm{MeV}$, we expect [10] the chiral partners of $B_{s}$ and $B_{s}^{*}$ to be $323 \mathrm{MeV}$ heavier, while the chiral partners of $B$ and $B^{*}$ to be $345 \mathrm{MeV}$ heavier, i.e. close to predictions in [13]. We note that any observation of chiral doubling for B mesons would be a strong validation for chiral doublers proposal. Indeed, in the recently proposed alternative scenarios discussed during this conference (multiquark states, hadronic molecules, modifications of quark potential, unitarization) a repeating pattern from charm to bottom calls for additional assumptions. 
Finally, let us mention very briefly the consequences of the chiral doublers scheme for the heavy light baryons, i.e. $h h l$ and $h l l$ type. Already in [1] we pointed, that the opposite parity baryons could be described as chiral solitons of the action of (3) type. In the light of presented here preliminary Selex data [7] and planned COMPASS experiment, the issue of doubly heavy baryons is no longer academic. It is tempting to speculate, that the observed splitting between the signals for the $c c u$ states of opposite parity, 3780 $\mathrm{MeV}$ and $3460 \mathrm{MeV}$ [7] is of chiral origin (here $340 \mathrm{MeV}$ ). Indeed, double heavy baryons, with the heavy diquark in spin 1 state behaving alike the heavy color source $\overline{3}_{c}$ resemble mesonic configurations and are subjected to chiral doubling due to the light quark physics, a point also noted recently by [13].

We do not discuss here to what extent the newly discovered chiral partners can shed more light on effects of charmonium absorption/regeneration in thermal models with medium effects. We stress that this is an important issue in light of the current and future experiments at RHIC and LHC as well as at GSI, probing the restoration of the chiral symmetry.

\section{ACKNOWLEDGMENTS}

This talk is based on work done in collaboration with Mannque Rho and Ismail Zahed [1, 10, 12]. This work was partially supported by the Polish State Committee for Scientific Research (KBN) grant 2P03B 09622 (2002-2004). I am very grateful to David Cassel, Murray Moinester, Jim Russ, Bob Cahn, Ken Hicks, Lonya Glozman, Pavel Krokovny, Kamal Seth, Ted Barnes, Dan-Olof Riska, Su Houng Lee, Maxim Polyakov, Zhenya Kolomeitsev, Thorsten Feldmann, Klaus Goeke, Frank Close and many others for passionate discussions on new particles during this conference.

\section{REFERENCES}

1. M.A. Nowak, M. Rho, I. Zahed, Phys. Rev. D48 (1993) 4370.

2. W. Bardeen and C. Hill, Phys. Rev. D49 (1994) 409.

3. BABAR Coll., B. Aubert et al., hep-ex/0304021

4. CLEO Coll., D. Besson et al., hep-ex/0305017 hep-ex/0305100

5. BELLE Coll., P. Krokovny et al., hep-ex/0308019

6. BABAR Coll., B. Aubert et al., hep-ex/0310050

7. James Russ and Murray Moinester, talks at Hadron03, Aschaffenburg, 31-08-06.09. 2003, Germany.

8. V.V. Kiselev, A. K. Likhoded, hep-ph/0103169

9. N. Isgur and M. B. Wise, Phys. Rev. Lett. 66 (1991) 1130. For early suggestions, see also E.V. Shuryak, Phys. Lett.93B (1980) 134, Nucl. Phys. B198 (1982) 83.

10. M.A. Nowak, M. Rho and I. Zahed, hep-ph/0307102

11. M.B. Wise, Phys. Rev. D45 (1992) R2118;. T.-M. Yan et al, Phys. Rev. D46 (1992) 1148; G. Burdman and J.F. Donoghue. Phys. Lett. B280 (1992) 287.

12. M.A. Nowak and I. Zahed, Phys. Rev. D48 (1993) 356.

13. W.A. Bardeen, E.J. Eichten, C.T. Hill, Phys. Rev. D68 (2003) 054024.

14. M. Musakhanov, hep-ph/0104163 and reference to unpublished results of P. Pobylitsa therein. 